\title{
Guest Editorial: Low-Voltage Integrated Circuits and Systems
}

\author{
Fabian Khateb ${ }^{1,2}$. Spyridon Vlassis ${ }^{3}$. \\ Tomasz Kulej ${ }^{4}$
}

Interest in developing new design techniques for low-voltage integrated circuits and systems has continued over several decades. It is mainly caused by system power constraints and advanced deep submicron technologies, which require lower and lower supply voltages. Since the early $90 \mathrm{~s}$, when the supply voltage of $3.3 \mathrm{~V}$ was considered as low, the required supply voltages have been constantly decreasing, in some applications dropping (much) below $1 \mathrm{~V}$. Nowadays, as predicted by the Technology Roadmap for Semiconductors, we can expect that the supply voltages will be reduced to $0.6 \mathrm{~V}$ for high performance microprocessors and $0.54 \mathrm{~V}$ for low-power solutions in a near future. Regarding the latter, in some cases even lower $V_{\mathrm{DD}}$ can be required, e.g., for circuits supplied with non-conventional energy sources, and devoted to some specific applications, like biomedical implants.

The increasing demand for both the low supply voltage and the energy efficiency often have a detrimental effect, especially upon analog and mixed signal circuits, which

\footnotetext{
$\otimes$ Fabian Khateb

khateb@feec.vutbr.cz

Spyridon Vlassis

svlassis@physics.upatras.gr

Tomasz Kulej

kulej@el.pcz.czest.pl

1 Department of Microelectronics, Brno University of Technology, Technická 10, Brno, Czech Republic

2 Faculty of Biomedical Engineering, Czech Technical University in Prague, nám. Sítná 3105, Kladno, Czech Republic

3 Electronics Laboratory, Physics Department, University of Patras, 26504, Rio, Achaia, Greece

4 Department of Electrical Engineering, Technical University of Częstochowa, 42-201 Częstochowa, Poland
} 
must operate with reduced voltage headroom and power dissipation. Therefore, the most challenging task for today's designers is to maintain the circuit performances by developing novel circuit structures capable of operating with highly reduced supply voltages. The main goal of this special issue is to present recent advances in the field and the main challenges relating to the theory, design and applications of low-voltage integrated circuits and systems. From 43 submitted manuscripts, a total of 15 papers have been accepted for publication. The papers are arranged to cover four main topics: digital circuits, analog functional blocks, RF circuits, supply and energy harvesting blocks. Below is a summary of the accepted contributions under each of the themes.

\section{Digital Circuits}

In the paper "High-speed digital domino logic for ultra-low supply voltages," a highspeed differential clocked voltage switch logic inverter operating at ultra-low supply voltages is presented. Simulated data for the new gate as well as preliminary measurements are presented. The increase in speed for supply voltages below $300 \mathrm{mV}$ is between 10 and 20 times compared to modified clocked voltage switch logic. In the paper "An Aging Aware Reliable FinFET-Based Low-Power 32 Word x 32-bit Register File," a reliable FinFET-based low-power 32 word x 32-bit register file in a $32 \mathrm{~nm}$ technology is introduced. A novel Aging aware dynamic OR gate is proposed here which is capable of maintaining a constant performance under negative bias temperature instability degradation. This proposed domino bit-line is further used to design a novel register file which is capable of maintaining a constant performance for a lifetime of more than 10 years.

\section{Analog Functional Blocks}

In the paper "A Low-Voltage PLL Design Using a New Calibration Technique for Low-Power Implantable Biomedical Systems," a low-voltage phase-locked loop (PLL) design using a new calibration technique for low-power implantable biomedical systems is described. The proposed PLL uses a switching technique in the charge pump and a bulk-driven technique in the voltage-controlled oscillator to reduce power consumption and operate at ultra-low voltage. In the paper "An ultra-low-energy analog comparator for A/D converters in CMOS image sensors," a new solution of an ultralow-energy analog comparator, dedicated to slope analog-to-digital converters (ADC), particularly suited for CMOS image sensors featuring a large number of ADCs is proposed. In the paper "Ultra-low-Voltage Integrable Electronic Realization of Integer and Fractional-order Liao's Chaotic Delayed Neuron Model," an ultra-low-voltage sinh-domain implementation of the neuron model is introduced. Moreover, for the first time, the fractional-order implementation of the model is presented. In the paper "Active current mirrors for low voltage analog circuit design," active current mirrors using sensing resistors and a pass transistor in a feedback loop are examined in detail. First, several non-idealities in this family of circuits like offset, noise, output impedance or bandwidth are addressed, showing no performance degradation under certain circumstances. Then, the design and measurement results of a $10 \mu \mathrm{A}$ (nominal) 
active current mirror that can operate down to just $80 \mathrm{mV}$ voltage drop are presented. In the paper "An Ultra-Low-Voltage Bulk-Driven Analog Voltage Buffer with Rail-toRail Input/Output Range," a low-voltage high driving capability rail-to-rail Class-AB CMOS bulk-driven operational transconductance amplifier (OTA) for analog voltage buffer with minimal current consumption is presented. The proposed OTA design consists of adaptively-biased Class-AB differential input stage to improve the effective transconductance and adaptive load relying on nonlinear current mirrors, configured in partial positive feedback mode to enhance the overall gain. In the paper "An accurate CMOS interface small capacitance variation sensing circuit for capacitive MEMS sensor applications," an accurate front-end CMOS interface circuit for sensing very small capacitance changes in capacitive sensors is presented. Finally, in the paper "Design and Analysis of an Ultra-Low-Power Second-Order Asynchronous Delta-Sigma Modulator," a second-order asynchronous delta-sigma modulator based on the active RC integrators and operating from supply voltage of $0.25 \mathrm{~V}$ is proposed. The OTAs used in the integrators are improved by employing a class- $\mathrm{AB}$ structure with bulk-driven input drivers and quasi-floating gate method in subthreshold region. These circuit approaches provide better linearity, signal-to-noise ratio and speed for the integrators, resulting in an overall improvement in the performance of the modulator.

\section{RF Circuits}

In the paper "Integrable CMOS-based Current-mode Sinusoidal Frequency and Peak Detector," the research proposes an integrable CMOS current-mode instantaneous frequency and peak detector (FPD) based on the trigonometric relationships and mathematic algorithm without the divider function and low-pass filter. The proposed FPD structure is straightforward, consisting of two differentiators, two multipliers and one square rooter. The FPD was experimentally utilized as the FM and AM demodulators with very satisfactory demodulation outcomes. In the paper "Multi-Objective Low Noise Amplifier Optimization Using Analytical Model and Genetic Computation," a methodology for multi-objective radio frequency (RF) low noise amplifier optimization using an analytical model of the MOS transistor in combination with genetic computation is introduced. In the paper "Analysis and Design of Single Reference Reduced Summer Loading based Switched Capacitor DFE," the design of a switched capacitor decision feedback equalizers for multi-tap post-cursor cancelation with reduced summer loading is addressed. The proposed architecture is validated by post-layout simulations.

\section{Supply and Energy Harvesting Blocks}

In the paper "A High-Efficiency CMOS Rectifier with Wide Harvesting Range and Wide Band Based on MPPT Technique for Low-Power IoT System Applications," a wide-harvesting-range, wide-band and high-efficiency CMOS rectifier for low-power application in Internet of Things systems is described. Through maximum power point tracking, the proposed rectifier can dynamically detect the output voltage to enable switching between various circuit modes in order to achieve higher power 
conversion efficiency, even during sub-1-V operation. In the paper "Design of FastLocked Digitally Controlled Low-Dropout Regulator for Ultra-low Voltage Input," a new design for a fast-locked digitally controlled low-dropout regulator for an ultralow voltage input is proposed. The proposed design involves a fast-locked control mechanism that reduces the settling time of the load transient response in the tracking mode and decreases the quiescent current in the regulating mode. In the paper "A $139 \mathrm{nW}, 67 \mathrm{ppm} /{ }^{\circ} \mathrm{C}$ BJT-CMOS-based voltage reference circuit," a low-power voltage reference circuit is developed using the principle that a thermal compensation of the threshold voltage of a diode-connected nMOSFET is obtained by using the PTAT current.

Acknowledgements We hope that all the papers presented in this special issue will be of interest to the readers. We would like to thank all the reviewers for their insightful comments, which helped us in evaluating the submitted papers. We also thank all the authors who have submitted their manuscripts for this special issue. Special thanks should be addressed to the Editor-in-Chief of the CSSP Journal, Prof. M.N.S. Swamy, for his valuable support and assistance during this project
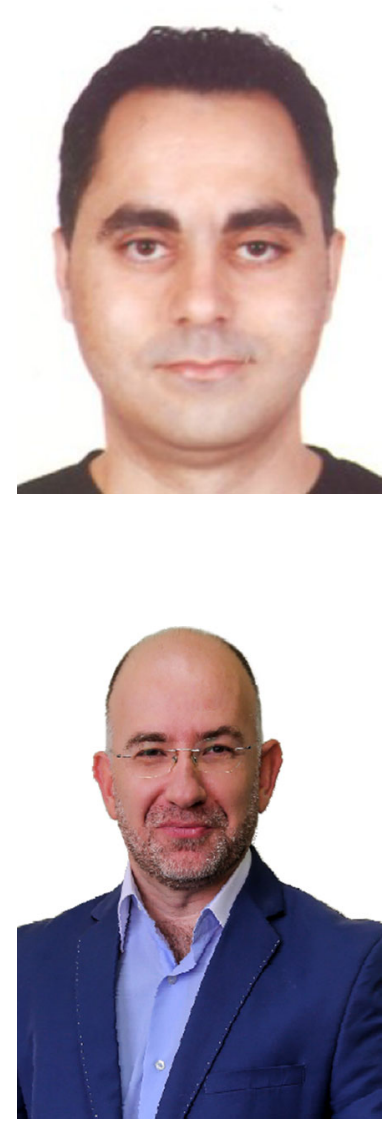

Fabian Khateb received the M.Sc. and Ph.D. degrees in Electrical Engineering and Communication and also in Business and Management from Brno University of Technology (BUT), Czech Republic in 2002, 2005, 2003 and 2007, respectively. He is currently working as Associate Professor at the Department of Microelectronics BUT and also at the Czech Technical University in Prague, Faculty of Biomedical Engineering, Joint Centre for Biomedical Engineering of Czech Technical University and Charles University in Prague. He has expertise in new principles of designing low-voltage low-power analog circuits, particularly biomedical applications. He is Associate Editor for Circuits, Systems and Signal Processing, and International Journal of Electronics. He is a member of the Editorial Board of IET Circuits, Devices \& Systems, and Microelectronics Journal. He holds four patents and he is author or co-author of more than 100 publications in journals and proceedings of international conferences.

Spyridon Vlassis received his B.Sc. (1994) in Physics, the M.Sc. (1996) in Electronics and Ph.D. (2000), all from Aristotle University of Thessaloniki, Greece. He has been working at senior level positions for established and VC-funded startup companies. During his carrier he has worked in the development of a) highperformance transceiver ICs in CMOS and BiCMOS processes for the $802.11 \mathrm{a} / \mathrm{b} / \mathrm{g} / \mathrm{j}$ WLAN markets b) MEMS based clock generator ICs and MEMS consumer applications and c) voltage regulators and extremely low-pass filters for CMOS transceiver ICs for hearing aids. He is currently Associate professor with the Electronics Laboratory, Department of Physics, University of Patras, Greece. He holds five patents and he has published over 70 papers in peer-reviewed journals and conferences. His research interests include ultra-low voltage analog IC design, biomedical circuit and systems, clock and data recovery and timing circuits for multi rate serial links. 


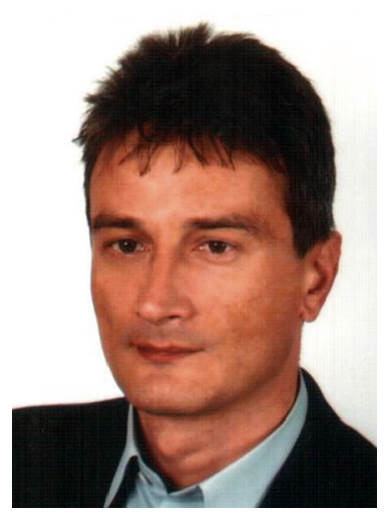

Tomasz Kulej received his M.Sc. and Ph.D. degrees from Gdańsk University of Technology, Gdńask, Poland in 1990 and 1996 respectively. He has considerable professional experience in the design and analysis of analog integrated circuits from both, industry and academia. Currently he is an Assistant Professor at the Department of Electrical Engineering, Częstochowa University of Technology, Częstochowa, Poland. His recent research interests include analog integrated circuits in CMOS technology, with emphasis to bulkdriven circuits. He is an author or co-author of over 50 publications in peer-reviewed journals and conferences and he holds two patents. 